\title{
Magneto-Optical Activity in Nonmagnetic Hyperbolic Nanoparticles
}

\author{
Joel Kuttruffø, ${ }^{1,2, *}$ Alessio Gabbani $\odot,{ }^{3, *}$ Gaia Petrucci $\odot,{ }^{3}$ Yingqi Zhao $\odot{ }^{4}$ Marzia Iarossi, ${ }^{4,5}$ \\ Esteban Pedrueza-Villalmanzo $\odot,{ }^{6,7}$ Alexandre Dmitriev $\odot,{ }^{6}$ Antonietta Parracino, ${ }^{8}$ Giuseppe Strangi, ${ }^{9,10}$ \\ Francesco De Angelis $\odot,{ }^{4}$ Daniele Brida $\odot,{ }^{1}$ Francesco Pineider $\odot,{ }^{3, \dagger}$ and Nicolò Maccaferri $\odot^{1,11, \$}$ \\ ${ }^{1}$ Department of Physics and Materials Science, University of Luxembourg, \\ 162 a avenue de la Faincerie, 1511, Luxembourg, Luxembourg \\ ${ }^{2}$ Department of Physics, University of Konstanz, Universitaetsstrasse 10, 78464 Konstanz, Germany \\ ${ }^{3}$ Dipartimento di Chimica e Chimica Industriale, Università di Pisa, Via Giuseppe Moruzzi 13, 56124, Pisa, Italy \\ ${ }^{4}$ Plasmon Nanotechnologies Unit, Istituto Italiano di Tecnologia, Via Morego 30, 16163, Genova, Italy \\ ${ }^{5}$ Dipartimento di Informatica, Bioingegneria, Robotica e Ingegneria dei Sistemi (DIBRIS). Università degli Studi di Genova, \\ Via Balbi 5, 16126 Genova, Italy \\ ${ }^{6}$ Department of Physics, University of Gothenburg, Universitetsplatsen 1, 405 30, Gothenburg, Sweden \\ ${ }^{7}$ Department of Chemistry and Chemical Engineering, Chalmers University of Technology, Kemigården 4, 41296 Göteborg, Sweden \\ ${ }^{8}$ Department of Chemistry, Uppsala University, Husargatan 3, 752 37, Uppsala, Sweden \\ ${ }^{9}$ Department of Physics, Case Western Reserve University, 10600 Euclid Avenue, 44106, Cleveland, Ohio, USA \\ ${ }^{10}$ CNR-NANOTEC Istituto di Nanotecnologia and Department of Physics, University of Calabria, \\ Via Pietro Bucci 87036, Rende, Italy \\ ${ }^{11}$ Department of Physics, Umeå University, Linnaeus väg 20, 90736 Umeå, Sweden
}

(Received 19 May 2021; accepted 23 September 2021; published 19 November 2021)

\begin{abstract}
Active nanophotonics can be realized by controlling the optical properties of materials with external magnetic fields. Here, we explore the influence of optical anisotropy on the magneto-optical activity in nonmagnetic hyperbolic nanoparticles. We demonstrate that the magneto-optical response is driven by the hyperbolic dispersion via the coupling of metallic-induced electric and dielectric-induced magnetic dipolar optical modes with static magnetic fields. Magnetic circular dichroism experiments confirm the theoretical predictions and reveal tunable magneto-optical activity across the visible and near infrared spectral range.
\end{abstract}

DOI: 10.1103/PhysRevLett.127.217402

Optical nanostructures enabling active control of light polarization are key components for future nanophotonic applications. One relevant example is the class of magnetophotonic nanomaterials which exploit magneto-optical (MO) enhancement produced by either pure metallic or pure dielectric materials [1]. Artificial anisotropic metamaterials combining metals and dielectrics enable more versatile lightmatter interaction engineering at the nanoscale [2,3], and their applications have diversified toward sensing [4,5], nanoscale waveguiding [6,7], enhanced nonlinearities [8-12], and amplified spontaneous emission [13-16]. The bulk optical properties of these materials can be described by an effective dielectric tensor, where the in-plane and out-ofplane permittivities, $\varepsilon_{\|}$and $\varepsilon_{\perp}$, respectively, can be almost arbitrarily tuned by the geometric dimension and material composition [17]. By stacking multiple layers of metal and dielectric materials, extreme optical anisotropies are

Published by the American Physical Society under the terms of the Creative Commons Attribution 4.0 International license. Further distribution of this work must maintain attribution to the author(s) and the published article's title, journal citation, and DOI. achieved, often described by hyperbolic isofrequency surfaces $\left(\varepsilon_{\|} \varepsilon_{\perp}<0\right)$, which are opposed to the elliptical ones observed in typical materials like pure metals or dielectrics. This optical anisotropy can be further exploited to enhance the MO response of a material [18-21], allowing to control the optical properties by applying an external magnetic field, which is fundamental in view of the development of active magnetophotonic devices [22-27], from sensing [28-32], and all-optical switching [33,34], to nonreciprocal light propagation [35-38] and polarization selection [39,40].

Here, we explore the fundamental coupling of an external magnetic field to the localized electric and magnetic modes excited in nonmagnetic type-II $\left(\varepsilon_{\|}<0, \varepsilon_{\perp}>0\right)$ hyperbolic nanoparticles, where full control of the optical properties has been recently demonstrated [41,42]. The broadband $\mathrm{MO}$ response of the sample is studied by magnetic circular dichroism (MCD) spectroscopy. We observe direct modulation of the MO response at both fundamental resonances of the system, which we, based on numerical modeling, interpret in terms of magnetic fieldinduced spatial confinement or broadening of circular currents in the nanoparticles. By developing an analytical framework based on quasi-isotropic Mie theory, we show 
that the MO response can be fundamentally described on the single particle level by considering the coupling of dipolar resonances to a static magnetic field.

Disk-shaped hyperbolic nanoparticles with a nominal diameter of $250 \mathrm{~nm}$ were fabricated on fused silica substrates using hole-mask colloidal lithography [43] (see Supplemental Material [44]). Two types of hybrid nanoparticles were prepared, in which the dielectric material is varied between $\mathrm{TiO}_{2}$ and $\mathrm{SiO}_{2}$ to allow for tuning of the fundamental absorption resonance [41]. Five alternating layers of $\mathrm{Au}$ $(10 \mathrm{~nm}$ each) and of the dielectric material $(20 \mathrm{~nm}$ each) were deposited. A SEM image of the $\mathrm{Au} / \mathrm{TiO}_{2}$ sample is shown in the inset of Fig. 1(a), alongside with the experimentally obtained optical extinction. Two peaks are clearly visible in the extinction spectrum, at $\lambda \sim 1000 \mathrm{~nm}$ and $\lambda \sim 1600 \mathrm{~nm}$. Since the nanoparticles are randomly distributed, we do not expect any collective effect and can consider the measured extinction as the average optical response of a single nanoparticle. The unusual optical properties of these nanoparticles can be described by an effective medium theory, where the anisotropy is represented by an in-plane permittivity $\varepsilon_{\|}$and an out-of-plane permittivity $\varepsilon_{\perp}$. These can be expressed as [17]

$$
\begin{gathered}
\varepsilon_{\|}=\frac{t_{m} \varepsilon_{m}+t_{d} \varepsilon_{d}}{t_{m}+t_{d}}, \\
\varepsilon_{\perp}=\frac{\varepsilon_{m} \varepsilon_{d}\left(t_{m}+t_{d}\right)}{t_{m} \varepsilon_{d}+t_{d} \varepsilon_{m}},
\end{gathered}
$$

where $t_{m(d)}$ is the thickness and $\varepsilon_{m(d)}$ is the permittivity of the metal (dielectric). The dispersion of the permittivity is shown as a function of the wavelength in the inset of Fig. 1(b). For $\lambda>500 \mathrm{~nm}, \varepsilon_{\perp}$ is positive (dielectriclike response) while $\varepsilon_{\|}$is negative (metal-like response), corresponding to a type-II hyperbolic dispersion relation. To validate our approach,
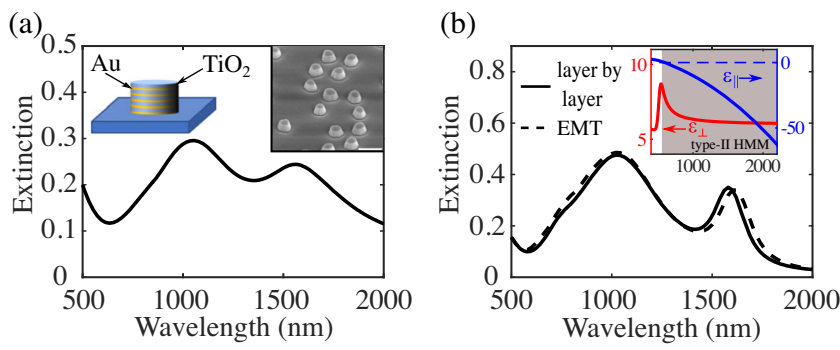

FIG. 1. (a) Experimental extinction spectrum of the $\mathrm{Au} / \mathrm{TiO}_{2}$ hyperbolic nanoparticle metasurface. Insets are a sketch of the geometry (top left) and a SEM image of the sample (top right, scale bar is $400 \mathrm{~nm}$ ). (b) Numerically obtained extinction spectra calculated based on the real structure (layer by layer, solid line) and using an effective medium theory (EMT, dashed line). Inset shows the real parts of the in-plane (blue) and out-of-plane (red) permittivities used in the EMT approach. For $\lambda>500 \mathrm{~nm}$, the system shows type-II hyperbolic dispersion (type-II HMM), as indicated in the figure (gray shaded area). full-wave numerical simulations have been performed using tabulated permittivity values for gold [45] (see also Supplemental Material [44]). First, the optical extinction was calculated considering the real structure with alternating layers [solid line in Fig. 1(b)]. Afterward, we considered a homogenized structure described by an anisotropic hyperbolic effective permittivity as expressed in Eqs. (1) and (2) [dashed line in Fig. 1(b)]. The resulting spectra are in excellent agreement and describe well the experimentally measured extinction, demonstrating that the physical origin of these modes lays on the hyperbolic nature of the system. It is worth noticing that the high energy plasmon decays mainly radiatively, while the low-energy plasmon decays mainly nonradiatively, as can be seen in Supplemental Material, Fig. 1, thanks to the ability of these architectures to separately tune both decay channels, in contrast with conventional plasmonic architectures [cf. plain gold nanodisks, Supplemental Material, Fig. 2(a)].

When a magnetic field is applied, the moving charges undergo a Lorentz force $\boldsymbol{F}_{\boldsymbol{L}}=q(\boldsymbol{v} \times \boldsymbol{B})$, oriented perpendicularly to the magnetic field and the trajectory of the charges. In this case, when a static field $H$ is applied along, for instance, the $z$ direction (out of plane), the symmetry of the dielectric function is broken, leading to nonzero off-diagonal components of the permittivity $\hat{\varepsilon}$ [50], i.e., (a)

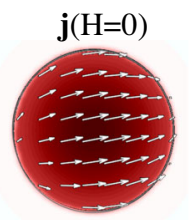

(b)

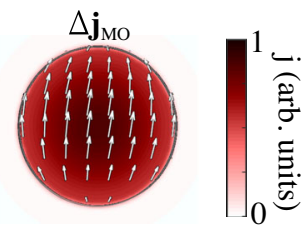

(c)

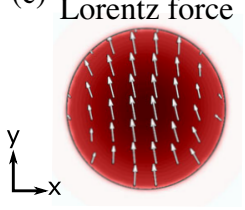

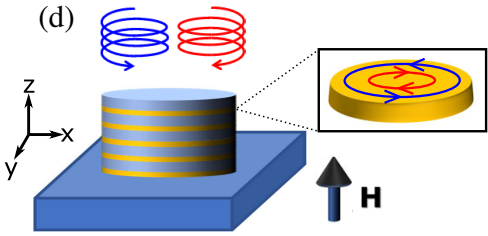

(e)
FIG. 2. (a)-(c) Calculated current density $\mathbf{j}$ (white arrows) at the low-energy resonance for zero magnetic field (a), magnetically induced change of current $\Delta \mathbf{j}_{\mathrm{MO}}(\mathrm{b})$, and Lorentz force calculated as cross product of $\mathbf{j}$ and the unit vector in direction of $\mathbf{H}$ (c). The absolute value of $j$ is color coded for all three cases. (d) Circular dichroism effect in terms of spatial confinement or broadening of circular modes. (e),(f) Induced relative change of current density $\Delta j / j$ (color coded) for opposite helicity of the incoming electric field, as sketched in the plot. Black arrows show the gradient of $\Delta j / j$ and display the induced spatial broadening or confinement of the circular plasmonic motion. 


$$
\hat{\varepsilon}=\left(\begin{array}{ccc}
\varepsilon_{x x} & \varepsilon_{\mathrm{MO}} & 0 \\
-\varepsilon_{\mathrm{MO}} & \varepsilon_{y y} & 0 \\
0 & 0 & \varepsilon_{z z}
\end{array}\right) .
$$

The nondiagonal MO term $\varepsilon_{\mathrm{MO}}$ is generally very small for noble metals compared to ferromagnetic materials (we assume $\varepsilon_{\mathrm{MO}}=10^{-4}+i 10^{-3}$, valid for $\mathrm{Au}$ at $1 \mathrm{~T}$, whereas $\varepsilon_{\mathrm{MO}}=1+i 0.1$ for Co [50]). However, nanoscale design of bulk materials can drastically alter the MO properties due to the presence of plasmon resonances, leading to an enhanced MO response [50-52]. The MO coupling is particularly interesting in the context of circularly polarized light, where dichroism is induced by the applied magnetic field in systems that otherwise exhibit rotational symmetry in the propagation direction of the incoming light [26]. When a static magnetic field is applied along the propagation direction, the degeneracy of left- and right-handed modes is lifted due to the off-diagonal coupling term in Eq. (3), which is observable by a derivativelike spectral shape of the MCD signal. In conventional plasmonic nanomaterials, this magnetically induced mode splitting is observed at the fundamental electric dipole resonance (as shown for plain gold nanodisks in Supplemental Material, Fig. 2) $[26,27]$. Here, by considering the coupling of the charges with the magnetic field, we predict MCD also at the low-energy magnetic dipole mode, which is related to the dielectric nature of the system, hence rendering this resonance magnetoactive and thereby enriching the versatility of the anisotropic system in view of active functionalities. We numerically assess the induced changes in the current density inside a single nanoparticle due to the interaction with an external magnetic field. All quantities are evaluated inside the topmost gold layer of the structure, which is resonantly excited at the low-energy magnetic dipole mode. For this case, the numerically calculated current density $\mathbf{j}$ at magnetic field $H=0$ is shown as white arrows in Fig. 2(a) for one phase of the incoming electric field together with the absolute value of the current density $j$ (color coded). Because of the MO coupling in the gold layers, when a static magnetic field is applied in positive or negative $z$ direction ( $+H$ and $-H$, respectively), we expect a magnetic-field dependent change in the current, $\Delta \dot{\boldsymbol{j}}_{\mathrm{MO}}=\frac{1}{2}[\boldsymbol{j}(+H)-\boldsymbol{j}(-H)][$ Fig. 2(b)]. Further, we directly compute the Lorentz force acting on the charges as the cross product of the current density and the magnetic field [Fig. 2(c)]. Indeed, the change in current density $\Delta j_{\text {MO }}$ follows well the direction of this force, as can be seen by comparing Figs. 2(b) and 2(c). One can notice orientation deviations of the arrows at the boundary of the particle, which is expected from the shape anisotropy of the system at its boundaries. An inversion of the magnetic field direction is topologically equivalent to a change in the helicity of the incoming light. Thus, for a fixed magnetic field and if averaged over one period of the electric field oscillation, following the sketch presented in Fig. 2(d), the magnetically induced force on the free charge carriers will lead to spatial confinement or broadening of the circular modes, strictly depending on the helicity of the incoming electric field. This is verified via the change in absolute (i.e., independent on the phase $\varphi$ ) current density $\Delta j$ in Figs. 2(e) and 2(f), and consequently results in a shift of the resonance frequency for opposite helicities (i.e., circular dichroism).

To experimentally probe the interaction of the circular modes with an external magnetic field, we use MCD spectroscopy [46,47] (see Supplemental Material [44]). We define the MCD via the difference between two extinction spectra acquired using light with opposite helicity, $E^{+}$(left-handed circular polarization) and $E^{-}$ (right-handed circular polarization) respectively, in the presence of an external magnetic field parallel to the incident light direction,

$$
\mathrm{MCD}=\frac{E^{+}-E^{-}}{E^{+}+E^{-}} .
$$

Corresponding experimental MCD spectra for the $\mathrm{Au} / \mathrm{TiO}_{2}$ (red diamonds) and $\mathrm{Au} / \mathrm{SiO}_{2}$ (yellow circles) samples are shown in Fig. 3(a). A derivativelike spectral shape is clearly observed at the low and the high energy resonances for the $\mathrm{Au} / \mathrm{TiO}_{2}$ sample. Such line shape is consistent with the excitation of circular plasmonic modes in noble metal nanostructures with rotational symmetry in the plane of the incident electric field, as we show for plain gold nanodisks in Supplemental Material, Fig. 2. For the hyperbolic nanoparticles, the same line shape of the MCD spectrum is observed for both the electric and magnetic dipole modes, in agreement with the rationalization reported above, i.e., magnetically induced spatial confinement or broadening of circular currents in the nanoparticles upon circularly polarized light excitation (Fig. 2). Interchanging the dielectric layer with $\mathrm{SiO}_{2}$ leads
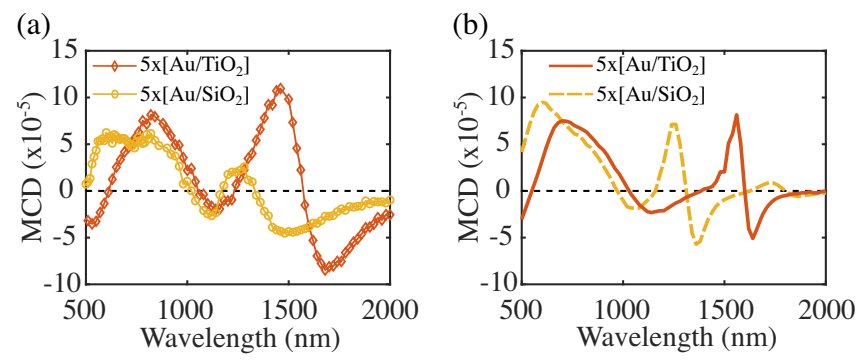

FIG. 3. (a) Experimental MCD spectra for the hyperbolic nanoparticles made of the two $\mathrm{Au} /$ dielectric multilayers: $5 x\left[\mathrm{Au} / \mathrm{TiO}_{2}\right]$ (red diamonds) and $5 x\left[\mathrm{Au} / \mathrm{SiO}_{2}\right]$ (yellow circles) samples acquired at $1.4 \mathrm{~T}$ applied magnetic field. (b) Simulated MCD spectra using the extended effective medium approach, including a $\mathrm{MO}$ coupling term, of the two $\mathrm{Au} /$ dielectric multilayers: $5 x\left[\mathrm{Au} / \mathrm{TiO}_{2}\right]$ (continuous line) and $5 x\left[\mathrm{Au} / \mathrm{SiO}_{2}\right]$ (dashed line). 
to a strong blueshift of the low-energy resonance, shown by the yellow circles in Fig. 3(a) (see also Supplemental Material, Fig. 1).

To describe the MO properties of our system in the framework of an effective medium theory, Eqs. (1) and (2) are generalized as

$$
\hat{\varepsilon}_{\mathrm{EMT}}=\left(\begin{array}{ccc}
\varepsilon_{\|} & f * \varepsilon_{M O} & 0 \\
-f * \varepsilon_{\mathrm{MO}} & \varepsilon_{\|} & 0 \\
0 & 0 & \varepsilon_{\perp}
\end{array}\right)
$$

where the off-diagonal component due to the coupling with the external magnetic field is considered for gold, and $f=1 / 3$ is the volume fraction of gold in the nanoparticle. Separate simulations are performed for both helicities and the MCD is calculated based on the individual extinction spectra according to Eq. (4). Resulting spectra are shown in Fig. 3(b) and reproduce well the observed spectral features. In these calculations, we assume a constant value of the MO coupling term, $\varepsilon_{\mathrm{MO}}=10^{-4}+i 10^{-3}$. Sepúlveda et al. further suggested a MO coupling term for the noble metals that is linear in the diagonal permittivity of the material, $\varepsilon_{\mathrm{MO}} \propto \varepsilon_{x x}$ [50]. This frequency dependent $\varepsilon_{\mathrm{MO}}(\omega)$ again reproduces the expected derivativelike spectral shape, however it overestimates the dichroism effect at the lowenergy resonance (Supplemental Material, Fig. 3).

The broadband MO response of the system fundamentally originates from the anisotropy of the dielectric function. The effect of this anisotropy is clearly observable in the numerically calculated electric near field of the resonantly excited nanoparticles (Supplemental Material, Fig. 4). At the high energy resonance, the major contribution originates from the in-plane electric field [Supplemental Material, Figs. 4(a) and 4(c)], while the out-of-plane contribution dominates for the low-energy resonance [Supplemental Material, Figs. 4(b) and 4(d)]. These results strongly suggest a dominant role of $\varepsilon_{\perp}$ and $\varepsilon_{\|}$ at the low and high energy resonances, respectively. To generalize this concept, we consider a spherical particle with radius $r$ displaying either isotropic permittivity $\varepsilon_{\perp}$ or $\varepsilon_{\|}$and calculate the scattering $\left(\sigma_{\text {sca }}\right)$ and absorption $\left(\sigma_{\text {abs }}\right)$ cross sections based on Mie theory [53,54]. The cross sections are calculated using a customized MATLAB code [48] as a weighted sum of the so-called Mie coefficients $a_{n}$ and $b_{n}$, describing dipolar $(n=1)$, quadrupolar $(n=2)$, etc., vector spherical harmonics of electric $\left(a_{n}\right)$ and magnetic $\left(b_{n}\right)$ nature [49]. The calculated Mie efficiencies $Q_{\mathrm{abs} / \mathrm{sca}}=\sigma_{\mathrm{abs} / \mathrm{sca}} / \pi r^{2}$ up to $n=1$ for $r=150 \mathrm{~nm}$ are presented in Figs. 4(a) and 4(b) for the in-plane and out-ofplane components of $\varepsilon$, respectively (for more details, see Supplemental Material [44]). To validate our approach, we compare the quasi-isotropic Mie theory with numerical results for a sphere with anisotropic permittivity $\hat{\varepsilon}=$ $\left(\varepsilon_{\|}, 0,0 ; 0, \varepsilon_{\|}, 0 ; 0,0, \varepsilon_{\perp}\right)$ (Supplemental Material, Fig. 5).
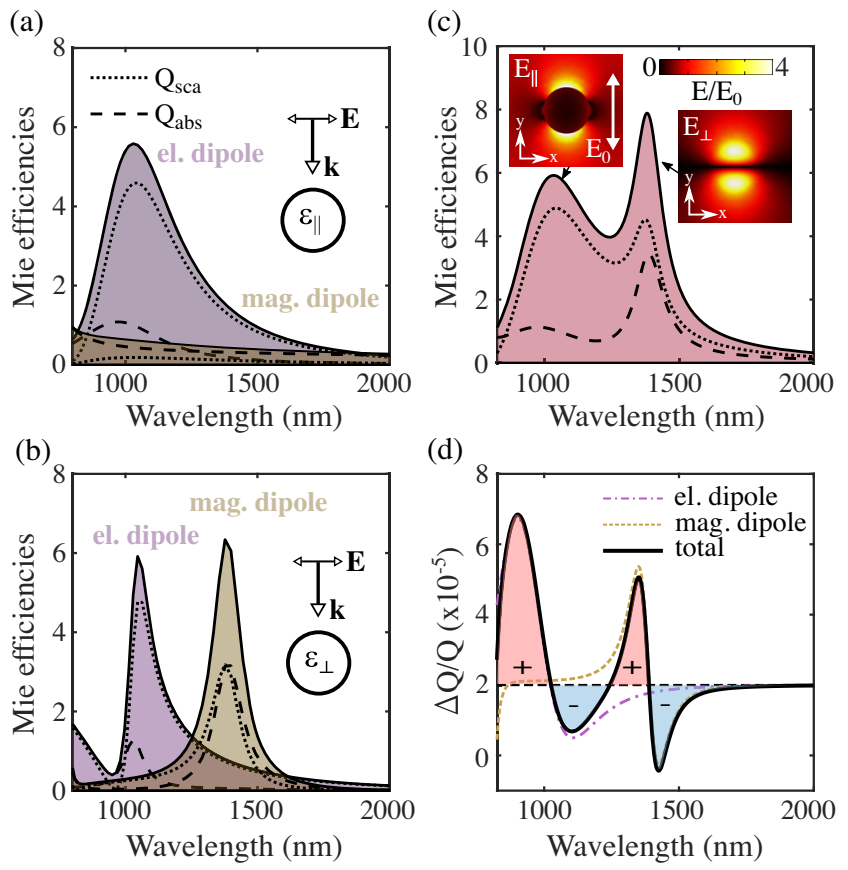

FIG. 4. Scattering (dotted lines) and absorption (dashed lines) efficiencies $Q_{\text {sca }}$ and $Q_{\text {abs }}$ for spherical nanoparticles with isotropic permittivity $\varepsilon_{\|}$(a) and $\varepsilon_{\perp}$ (b). Interaction efficiencies are analytically calculated with the Mie expansion up to the first order, where magnetic (green) and electric (purple) contributions are separately evaluated. (c) Sum of the in-plane electric dipole shown in (a) and the out-of-plane magnetic dipole shown in (b). Inset are near-field plots of the resonances normalized to the incoming field $E_{0}$ indicated by the white arrow. (d) Differential extinction spectrum $\Delta Q / Q$ calculated from the magnetically induced dichroism effect. Contributions from the electric and magnetic dipoles are marked by the dash-dotted and dashed lines, respectively. The overall spectrum (black solid line) exhibits a derivative spectral shape at the resonances, as indicated by the light blue and light red areas under the curve.

As for the hyperbolic disks, the numerical extinction shows high and low-energy resonances which we can now allocate to an in-plane electric and an out-of-plane magnetic dipole, respectively. In our quasi-isotropic model, higher absorption at the low-energy resonance can be accounted for by adding a small fraction of the imaginary part of $\varepsilon_{m}$ to Eq. (2) (see parametric study in Supplemental Material, Fig. 6). The out-of-plane electric dipole is omitted here, because the in-plane permittivity contributes dominantly at that wavelength (Supplemental Material, Fig. 4). The sum of in-plane electric dipole and out-of-plane magnetic dipole is shown in Fig. 4(c) and agrees well with the numerical results (Supplemental Material, Fig. 5).

For noble metals like gold, the MO coupling is predominantly determined by free carriers in a broad spectral range [50] and the optical properties of the metal conduction electrons are well described by the Drude model. We consider the coupling of these charges to an external magnetic field in a perturbative approach [55], i.e., we 
introduce a magnetic-field-dependent dielectric function of the metal, $\varepsilon_{m}=\varepsilon_{m}^{0} \pm \varepsilon_{\mathrm{MCD}}(H)$. Since a change in the helicity of the incoming light is topologically equivalent to an inversion of the magnetic field direction, we can also understand the MCD in terms of a magnetically induced change of the plasmonic resonance condition for a fixed polarization of the incoming light. In our perturbative approach, we can express their MO coupling as [23]

$$
\varepsilon_{\mathrm{MCD}}(H)=\frac{-2 \omega_{c}(H) \omega_{p}^{2} \tau^{3}}{\left[\omega_{c}(H)^{2} \tau^{2}-\tau^{2} \omega^{2}+1\right]^{2}+4 \tau^{2} \omega^{2}},
$$

where $\tau$ is the electron relaxation time, $\omega_{p}^{2}=N e^{2} / \varepsilon_{0} m^{*}$ the plasma frequency, and $\omega_{c}(H)=e H / m^{*}$ the magneticfield-dependent cyclotron frequency ( $e$ is the electron charge, $m^{*}$ the effective mass, and $N$ the electron density). Inverting the applied magnetic field will thus change the orbital frequency of the free carriers, leading to a $H$-dependent modification of the plasmonic resonance condition, resulting in a spectral shift of the mode for an applied magnetic field. We can then express the induced MCD via the change in extinction caused by inversion of the magnetic field direction as

$$
\frac{\Delta Q}{Q}=\frac{Q^{(+H)}-Q^{(-H)}}{Q^{(H=0)}}
$$

where $Q^{(H=0)}$ is the extinction efficiency at $H=0$. The corresponding spectrum is shown in Fig. 4(d) (black solid line). From the Mie expansion of the extinction, we can distinguish the contributions to the dichroism coming from either the electric (dash-dotted line) or the magnetic (dashed line) dipole resonance, thus obtaining a derivativelike spectral shape of the differential spectrum at both resonances, which describes well the induced dichroism observed in our experiments.

Finally, it is worth noticing that this system can be directly used also for active control of the polarization state of light via the polar MO Kerr effect without losing on modulation strength. Additionally, we can expect MO coupling in the longitudinal configuration, where the response should be one order of magnitude less strong. However, in the latter case nontrivial coupling between the different resonances might provide additional enhancement [35]. As well, we expect that all these MO effects can be enhanced by at least two orders of magnitude if the metallic building block is a magnetic material. Moreover, if the metallic layer is made of $\mathrm{Co} / \mathrm{Pt}$ or $\mathrm{Au} / \mathrm{Co}$ multilayers, this will help in reducing the applied field by almost one order of magnitude due to the strong magnetic anisotropy promoting magnetization along the out-of-plane direction [56,57]. Alternatively, the use of magnetic garnets as dielectric building blocks can also boost the MO response of these nanoparticles, as was previously demonstrated in iron garnets doped with gold nanoparticles and multilayered magnetoplasmonic crystals [58-60]. Although the quality factor of the resonances reported in this work is not high and will further decrease when magnetic materials are employed, it was recently demonstrated for pure plasmonic nanoparticles that this factor can be improved, even by orders of magnitude compared to that of the constituent build blocks, by ordering the particles in a crystal-like structure and playing with their size and shape [61]. This approach will add interesting perspectives on how to combine coherent interparticle coupling, such as for surface lattice resonances, with anisotropic optical dispersion.

In conclusion, we experimentally and theoretically demonstrated MO activity in type-II hyperbolic nanoparticles by reasonably low $(\sim 1 \mathrm{~T})$ external magnetic fields. Numerical simulations support the experimental results and prove the viability of the effective medium theory for the description of the anisotropic permittivity of these nanoparticles. Moreover, numerical modeling allows addressing the $\mathrm{MO}$ coupling in terms of induced changes in the current density of the system. We established a Mie-theory-based analytical model, calculating the MO response by considering the interaction of a static magnetic field with hyperbolic dispersion-induced magnetic and electric dipolar modes in individual nanoparticles. Beyond fundamental insights on the physics of the system, this analytical approach is general and can be expanded to other geometries and materials. Our results open exciting opportunities for the development of novel nanophotonic active materials combining the benefits of subwavelength light manipulation with the ability to freely tune the MO response via control over the anisotropy in the permittivity of the system.

N.M. acknowledges support from the Luxembourg National Research Fund (Grant No. C19/MS/13624497 "ULTRON") and the European Union under the FETOPEN-01-2018-2019-2020 call (Grant No. 964363 "ProID"). D. B. acknowledge support from the European Research Council (Grant No. 819871 "UpTEMPO"). D. B. and N. M. acknowledge support from the ERDF Program (Grant No. 2017-03-022-19 'Lux-Ultra-Fast'). A. G., G. P., E. P. V., A. D., and F. P. acknowledge the financial support of H2020 FETOPEN-2016-2017 Grant No. 737709 FEMTOTERABYTE (E. C.). A. G., G. P., and F.P. acknowledge the financial support of Italian MIUR through PRIN 2017 (project Q-ChiSS). J. K. acknowledges support from the Deutsche Forschungsgemeinschaft (DFG, Project No. 425217212, SFB 1432).

\footnotetext{
*These authors contributed equally to this work. †rancesco.pineider@unipi.it †nicolo.maccaferri@uni.lu nicolo.maccaferri@umu.se

[1] N. Maccaferri, I. Zubritskaya, I. Razdolski, I.-A. Chioar, V. Belotelov, V. Kapaklis, P. M. Oppeneer, and A. Dmitriev,
} 
Nanoscale magnetophotonics, J. Appl. Phys. 127, 080903 (2020).

[2] A. Poddubny, I. Iorsh, P. Belov, and Y. Kivshar, Hyperbolic metamaterials, Nat. Photonics 7, 948 (2013).

[3] I. I. Smolyaninov, Hyperbolic metamaterial geometries and basic properties, in Hyperbolic Metamaterials (Morgan \& Claypool Publishers, San Rafael, 2018), https://dx.doi.org/ 10.1088/978-1-6817-4565-7.

[4] K. V. Sreekanth, Y. Alapan, M. ElKabbash, E. Ilker, M. Hinczewski, U. A. Gurkan, A. De Luca, and G. Strangi, Extreme sensitivity biosensing platform based on hyperbolic metamaterials, Nat. Mater. 15, 621 (2016).

[5] A. Carrara, N. Maccaferri, A. Cerea, A. Bozzola, F. De Angelis, R. Proietti Zaccaria, and A. Toma, Plasmon hybridization in compressible metal-insulator-metal nanocavities: An optical approach for sensing deep sub-wavelength deformation, Adv. Opt. Mater. 8, 2000609 (2020).

[6] J. A. Dionne, L. A. Sweatlock, H. A. Atwater, and A. Polman, Planar metal plasmon waveguides: Frequencydependent dispersion, propagation, localization, and loss beyond the free electron model, Phys. Rev. B 72, 075405 (2005).

[7] V. Caligiuri, A. Pianelli, M. Miscuglio, A. Patra, N. Maccaferri, R. Caputo, and A. De Luca, Near- and mid-infrared graphenebased photonic architectures for ultrafast and low-power electro-optical switching and ultra-high resolution imaging, ACS Appl. Nano Mater. 3, 12218 (2020).

[8] W. Wenyang, L. Fan, W. Zang, X. Yang, P. Zhan, Z. Chen, and Z. Wang, Second harmonic generation enhancement from a nonlinear nanocrystal integrated hyperbolic metamaterial cavity, Opt. Express 25, 21342 (2017).

[9] S. Kruk, M. Weismann, A. Yu. Bykov, E. A. Mamonov, I. A. Kolmychek, T. Murzina, N. C. Panoiu, D. N. Neshev, and Y.S. Kivshar, Enhanced magnetic second-harmonic generation from resonant metasurfaces, ACS Photonics 2, 1007 (2015).

[10] S. Suresh, O. Reshef, M. Z. Alam, J. Upham, M. Karimi, and R. W. Boyd, Enhanced nonlinear optical responses of layered epsilon-near-zero metamaterials at visible frequencies, ACS Photonics 8, 125 (2021).

[11] J. Kuttruff, D. Garoli, J. Allerbeck, R. Krahne, A. De Luca, D. Brida, V. Caligiuri, and N. Maccaferri, Ultrafast alloptical switching enabled by epsilon-near-zero-tailored absorption in metal-insulator nanocavities, Commun. Phys. 3, 114 (2020).

[12] N. Maccaferri, A. Zilli, T. Isoniemi, L. Ghirardini, M. Iarossi, M. Finazzi, M. Celebrano, and F. De Angelis, Enhanced nonlinear emission from single multilayered metal-dielectric nanocavities resonating in the near-infrared, ACS Photonics 8, 512 (2021).

[13] S. R. K. C. Indukuri, J. Bar-David, N. Mazurski, and U. Levy, Ultrasmall mode volume hyperbolic nanocavities for enhanced light-matter interaction at the nanoscale, ACS Nano 13, 11770 (2019).

[14] D. Lu, H. Qian, K. Wang, H. Shen, F. Wei, Y. Jiang, E. E. Fullerton, P. K. L. Yu, and Z. Liu, Nanostructuring multilayer hyperbolic metamaterials for ultrafast and bright green InGaN quantum wells, Adv. Mater. 30, 1706411 (2018).

[15] S. R. K. C. Indukuri, C. Frydendahl, J. Bar-David, N. Mazurski, and U. Levy, $\mathrm{WS}_{2}$ monolayers coupled to hyperbolic metamaterial nanoantennas: Broad implications for light-matter-interaction applications, ACS Appl. Nano Mater. 3, 10226 (2020).

[16] D. Lu, J. J. Kan, E. E. Fullerton, and Z. Liu, Enhancing spontaneous emission rates of molecules using nanopatterned multilayer hyperbolic metamaterials, Nat. Nanotechnol. 9, 48 (2014).

[17] V. Caligiuri, R. Dhama, K. V. Sreekanth, G. Strangi, and A. De Luca, Dielectric singularity in hyperbolic metamaterials: The inversion point of coexisting anisotropies, Sci. Rep. 6, 20002 (2016).

[18] X. Wang, H. Wang, J. Jian, B. X. Rutherford, X. Gao, X. $\mathrm{Xu}$, and $\mathrm{H}$. Wang, Metal-free oxide-nitride heterostructure as a tunable hyperbolic metamaterial platform, Nano Lett. 20, 6614 (2020).

[19] I. A. Kolmychek, A. R. Pomozov, A. P. Leontiev, K. S. Napolskii, and T. V. Murzina, Magneto-optical effects in hyperbolic metamaterials, Opt. Lett. 43, 3917 (2018).

[20] I. V. Malysheva, I. A. Kolmychek, A. M. Romashkina, A. P. Leontiev, K. S. Napolskii, and T. V. Murzina, Magnetooptical effects in hyperbolic metamaterials based on ordered arrays of bisegmented gold/nickel nanorods, Nanotechnology 32, 305710 (2021).

[21] B. Fan, M. E. Nasir, L. H. Nicholls, A. V. Zayats, and V. A. Podolskiy, Magneto-optical metamaterials: Nonreciprocal transmission and Faraday effect enhancement, Adv. Opt. Mater. 7, 1801420 (2019).

[22] A. López-Ortega, M. Zapata-Herrera, N. Maccaferri, M. Pancaldi, M. Garcia, A. Chuvilin, and P. Vavassori, Enhanced magnetic modulation of light polarization exploiting hybridization with multipolar dark plasmons in magnetoplasmonic nanocavities, Light Sci. Appl. 9, 49 (2020).

[23] G. Armelles, A. Cebollada, A. García-Martín, and M. U. González, Magnetoplasmonics: Combining magnetic and plasmonic functionalities, Adv. Opt. Mater. 1, 10 (2013).

[24] N. Maccaferri, Coupling phenomena and collective effects in resonant meta-atoms supporting both plasmonic and (opto-)magnetic functionalities: An overview on properties and applications, J. Opt. Soc. Am. B 36, E112 (2019).

[25] D. O. Ignatyeva, D. Karki, A. A. Voronov, M. A. Kozhaev, D. M. Krichevsky, A. I. Chernov, M. Levy, and V. I. Belotelov, All-dielectric magnetic metasurface for advanced light control in dual polarizations combined with high- $Q$ resonances, Nat. Commun. 11, 5487 (2020).

[26] F. Pineider, G. Campo, V. Bonanni, C. de Julián Fernández, G. Mattei, A. Caneschi, and C. Sangregorio, Circular magnetoplasmonic modes in gold nanoparticles, Nano Lett. 13, 4785 (2013).

[27] A. Gabbani, E. Fantechi, G. Petrucci, G. Campo, C. de Julián Fernández, P. Ghigna, L. Sorace, V. Bonanni, M. Gurioli, C. Sangregorio, and F. Pineider, Dielectric effects in FeO $x$-coated Au nanoparticles boost the magnetoplasmonic response: Implications for active plasmonic devices, ACS Appl. Nano Mater. 4, 1057 (2021).

[28] N. Maccaferri, K. E. Gregorczyk, T. V. A. G. de Oliveira, M. Kataja, S. van Dijken, Z. Pirzadeh, A. Dmitriev, J. Åkerman, M. Knez, and P. Vavassori, Ultrasensitive and label-free molecular-level detection enabled by light phase control in magnetoplasmonic nanoantennas, Nat. Commun. 6, 6150 (2015). 
[29] B. F. Diaz-Valencia, J. R. Mejía-Salazar, O. N. Oliveira Jr., N. Porras-Montenegro, and P. Albella, Enhanced transverse magneto-optical Kerr effect in magnetoplasmonic crystals for the design of highly sensitive plasmonic (bio)sensing platforms, ACS Omega 2, 7682 (2017).

[30] V. T. Tran, J. Kim, L. T. Tufa, S. Oh, J. Kwon, and J. Lee, Magnetoplasmonic nanomaterials for biosensing/imaging and in vitro/in vitro biousability, Anal. Chem. 90, 225 (2018).

[31] D. O. Ignatyeva, P. O. Kapralov, G. A. Knyazev, S. K. Sekatskii, G. Dietler, M. Nur-E-Alam, M. Vasiliev, K. Alameh, and V.I. Belotelov, High- $Q$ surface modes in photonic crystal/iron garnet film heterostructures for sensor applications, JETP Lett. 104, 679 (2016).

[32] M. G. Manera, A. Colombelli, A. Taurino, A. GarcíaMartín, and R. Rella, Magneto-optical properties of noble-metal nanostructures: Functional nanomaterials for bio sensing, Sci. Rep. 8, 12640 (2018).

[33] D. O. Ignatyeva, C. S. Davies, D. A. Sylgacheva, A. Tsukamoto, H. Yoshikawa, P. O. Kapralov, A. Kirilyuk, V. I. Belotelov, and A. V. Kimel, Plasmonic layer-selective all-optical switching of magnetization with nanometer resolution, Nat. Commun. 10, 4786 (2019).

[34] I. A. Novikov, M. A. Kiryanov, P. K. Nurgalieva, A. Yu. Frolov, V. V. Popov, T. V. Dolgova, and A. A. Fedyanin, Ultrafast magneto-optics in nickel magnetoplasmonic crystals, Nano Lett. 20, 8615 (2020).

[35] K. Lodewijks, N. Maccaferri, T. Pakizeh, R. K. Dumas, I. Zubritskaya, J. Åkerman, P. Vavassori, and A. Dmitriev, Magnetoplasmonic design rules for active magneto-optics, Nano Lett. 14, 7207 (2014).

[36] J. Y. Chin, T. Steinle, T. Wehlus, D. Dregely, T. Weiss, V. I. Belotelov, B. Stritzker, and H. Giessen, Nonreciprocal plasmonics enables giant enhancement of thin-film Faraday rotation, Nat. Commun. 4, 1599 (2013).

[37] D. Floess, M. Hentschel, T. Weiss, H.-U. Habermeier, J. Jiao, S. G. Tikhodeev, and H. Giessen, Plasmonic Analog of Electromagnetically Induced Absorption Leads to Giant Thin Film Faraday Rotation of $14^{\circ}$, Phys. Rev. X 7, 021048 (2017).

[38] D. Floess and H. Giessen, Nonreciprocal hybrid magnetoplasmonics, Rep. Prog. Phys. 81, 116401 (2018).

[39] F. Wu, G. Lu, Z. Guo, H. Jiang, C. Xue, M. Zheng, C. Chen, G. Du, and H. Chen, Redshift Gaps in One-Dimensional Photonic Crystals Containing Hyperbolic Metamaterials, Phys. Rev. Applied 10, 064022 (2018).

[40] S. S. Kruk, C. Helgert, M. Decker, I. Staude, C. Menzel, C. Etrich, C. Rockstuhl, C. Jagadish, T. Pertsch, D. N. Neshev, and Y.S. Kivshar, Optical metamaterials with quasicrystalline symmetry: Symmetry-induced optical isotropy, Phys. Rev. B 88, 201404(R) (2013).

[41] N. Maccaferri, Y. Zhao, T. Isoniemi, M. Iarossi, A. Parracino, G. Strangi, and F. De Angelis, Hyperbolic meta-antennas enable full control of scattering and absorption of light, Nano Lett. 19, 1851 (2019).

[42] T. Isoniemi, N. Maccaferri, Q. M. Ramasse, G. Strangi, and F. De Angelis, Electron energy loss spectroscopy of bright and dark modes in hyperbolic metamaterial nanostructures, Adv. Opt. Mater. 8, 2000277 (2020).
[43] H. Fredriksson, Y. Alaverdyan, A. Dmitriev, C. Langhammer, D. S. Sutherland, M. Zäch, and B. Kasemo, Hole-mask colloidal lithography, Adv. Mater. 19, 4297 (2007).

[44] See Supplemental Material at http://link.aps.org/supplemental/ 10.1103/PhysRevLett.127.217402 for more details about samples fabrication, which includes Ref. [43]; more details about numerical simulations, which includes Ref. [45]; more details about experimental measurements, which includes Refs. [46,47]more details about the analytical calculations, which includes Refs. [48,49].

[45] A. Rakić, A. Djurišić, J. Elazar, and M. Majewski, Optical properties of metallic films for vertical-cavity optoelectronic devices, Appl. Opt. 37, 5271.

[46] B. Han, X. Gao, J. Lv, and Z. Tang, Magnetic circular dichroism in nanomaterials: New opportunity in understanding and modulation of excitonic and plasmonic resonances, Adv. Mater. 32, 1801491 (2020).

[47] W. R. A. Mason, A Practical Guide to Magnetic Circular Dichroism Spectroscopy (Wiley-Interscience, New York, 2007).

[48] C. Matzler, MATLAB functions for Mie scattering and absorption, IAP Res. Rep. No. 2002-11 2002.

[49] C. F. Bohren and D. R. Huffman, Absorption and Scattering of Light by Small Particles (John Wiley \& Sons, New York, 2008).

[50] B. Sepúlveda, J. B. González-Díaz, A. García-Martín, L. M. Lechuga, and G. Armelles, Plasmon-Induced MagnetoOptical Activity in Nanosized Gold Disks, Phys. Rev. Lett. 104, 147401 (2010).

[51] N. Maccaferri, A. Berger, S. Bonetti, V. Bonanni, M. Kataja, Q. H. Qin, S. van Dijken, Z. Pirzadeh, A. Dmitriev, J. Nogués, J. Åkerman, and P. Vavassori, Tuning the MagnetoOptical Response of Nanosize Ferromagnetic Ni Disks Using the Phase of Localized Plasmons, Phys. Rev. Lett. 111, 167401 (2013).

[52] N. Maccaferri, M. Kataja, V. Bonanni, S. Bonetti, Z. Pirzadeh, A. Dmitriev, S. van Dijken, J. Åkerman, and P. Vavassori, Effects of a non-absorbing substrate on the magneto-optical Kerr response of plasmonic ferromagnetic nanodisks, Phys. Status Solidi (a) 211, 1067 (2014).

[53] G. Mie, Beiträge zur optik trüber medien, speziell kolloidaler metallösungen, Ann. Phys. (Berlin) 330, 377 (1908).

[54] M. K. Schmidt, R. Esteban, J. Sáenz, I. Suárez-Lacalle, S. Mackowski, and J. Aizpurua, Dielectric antennas - A suitable platform for controlling magnetic dipolar emission, Opt. Express 20, 13636 (2012).

[55] Y. Gu and K. G. Kornev, Plasmon enhanced direct and inverse Faraday effects in nonmagnetic nanocomposites, J. Opt. Soc. Am. B 27, 2165 (2010).

[56] J. Wang, T. Sannomiya, J. Shia, and Y. Nakamura, Perpendicular magnetic anisotropy and perpendicular exchange bias in sputter-deposited $\mathrm{CoO} / \mathrm{CoPt}$ multilayer, J. Appl. Phys. 113, 17D714 (2013).

[57] C. Rizal and E. E. Fullerton Perpendicular magnetic anisotropy and microstructure properties of nanoscale Co/Au multilayers, J. Phys. D 50, 355002 (2017).

[58] S. Tomita, T. Kato, S. Tsunashima, S. Iwata, M. Fujii, and S. Hayashi Magneto-Optical Kerr Effects of Yttrium-Iron Garnet Thin Films Incorporating Gold Nanoparticles, Phys. Rev. Lett. 96, 167402 (2006). 
[59] V. I. Belotelov, I. A. Akimov, M. Pohl, V. A. Kotov, S. Kasture, A. S. Vengurlekar, A. Venu Gopal, D. R. Yakovlev, A. K. Zvezdin, and M. Bayer, Enhanced magneto-optical effects in magnetoplasmonic crystals, Nat. Nanotechnol. 6, 370 (2011).

[60] S. D. Pappas, P. Lang, T. Eul, M. Hartelt, A. García-Martín, B. Hillebrands, M. Aeschlimann, and E. Th. Papaioannou, Near-field mechanism of the enhanced broadband magneto-optical activity of hybrid $\mathrm{Au}$ loaded Bi:YIG, Nanoscale 12, 7309 (2020).

[61] M. S. Bin-Alam, O. Reshef, Y. Mamchur, M. Z. Alam, G. Carlow, J. Upham, B. T. Sullivan, J.-M. Ménard, M. J. Huttunen, R. W. Boyd, and K. Dolgaleva, Ultra-high- $Q$ resonances in plasmonic metasurfaces, Nat. Commun. 12, 974 (2021). 